\title{
Retrieval of Atmospheric Attenuation Using Combined Ground-Based and Airborne 95-GHz Cloud Radar Measurements
}

\author{
Lihua Li, Stephen M. Sekelsky, Steven C. Reising, and Calvin T. Swift \\ Microwave Remote Sensing Laboratory, University of Massachusetts at Amherst, Amherst, Massachusetts \\ Stephen L. Durden, Gregory A. Sadowy, Steven J. Dinardo, and Fuk K. Li \\ Jet Propulsion Laboratory, California Institute of Technology, Pasadena, California \\ Arlie Huffman and Graeme Stephens \\ Department of Atmospheric Science, Colorado State University, Fort Collins, Colorado \\ DAVID M. BABB AND HANS W. ROSENBERGER \\ Department of Meteorology, The Pennsylvania State University, University Park, Pennsylvania
}

(Manuscript received 31 January 2000, in final form 25 September 2000)

\begin{abstract}
Cloud measurements at millimeter-wave frequencies are affected by attenuation due to atmospheric gases, clouds, and precipitation. Estimation of the true equivalent radar reflectivity, $Z_{e}$, is complicated because extinction mechanisms are not well characterized at these short wavelengths. This paper discusses cloud radar calibration and intercomparison of airborne and ground-based radar measurements and presents a unique algorithm for attenuation retrieval. This algorithm is based on dual $95-\mathrm{GHz}$ radar measurements of the same cloud and precipitation volumes collected from opposing viewing angles. True radar reflectivity is retrieved by combining upward-looking and downward-looking radar profiles. This method reduces the uncertainty in radar reflectivity and attenuation estimates, since it does not require a priori knowledge of hydrometeors' microphysical properties. Results from this technique are compared with results retrieved from the Hitschfeld and Bordan algorithm, which uses single-radar measurements with path-integrated attenuation as a constraint. Further analysis is planned to employ this dual-radar algorithm in order to refine single-radar attenuation retrieval techniques, which will be used by operational sensors such as the CloudSat radar.
\end{abstract}

\section{Introduction}

Understanding the effects of clouds on the earth's radiation budget is essential to research on climate change. The lack of finescale cloud data is apparent in current climate model simulations (Houghton et al. 1995). For cloud observations, millimeter-wave radars offer significant advantages over low-frequency radars (Lhermitte 1987; Mead et al. 1994; Sekelsky and McIntosh 1996; Clothiaux et al. 1995). Because of the short wavelengths and the high-scattering efficiency of hydrometeors at millimeter-wave frequencies, compact low-power radars can be built for use in portable systems, especially for airborne (Pazmany et al. 1994; Sadowy et al. 1997) and spaceborne applications. Re-

Corresponding author address: Dr. Lihua Li, Microwave Remote Sensing Laboratory, University of Massachusetts at Amherst, Knowles Bldg., Room 201, Amherst, MA 01003.

E-mail: lihua@mirsl.ecs.umass.edu cently, a 94-GHz spaceborne cloud radar proposed by the National Aeronautics and Space Administration (NASA; Li et al. 1994) for the CloudSat program has received funding. One of the challenges of using millimeter-wave radar for quantitative cloud measurements is quantifying attenuation because attenuation due to atmospheric gases, clouds, and precipitation is considerable at these frequencies, especially when precipitation or water clouds are within the radar signal propagation path. For single radar measurements, retrieval of the true radar reflectivity under attenuating conditions is not trivial. It requires knowledge of the microphysical characteristics of the hydrometeors. In addition, although attenuation is an unwanted effect, its measurement provides valuable information about the properties of the hydrometeors.

In order to better characterize atmospheric attenuation at $95-\mathrm{GHz}$ (W band) and to provide an opportunity for calibration comparison among active research radars 
TABLE 1. The ACR and CPRS W-band radar system parameters.

\begin{tabular}{lll}
\hline \hline & \multicolumn{1}{c}{ CPRS } \\
& W band & \multicolumn{1}{c}{ ACR } \\
\hline Frequency $(\mathrm{GHz})$ & 94.92 & $\begin{array}{c}94.905,94.915, \\
94.925,94.935\end{array}$ \\
Transmit polarization & V or H & V or H \\
Receive polarization & V and $\mathrm{H}$ & V or H \\
Peak power $(\mathrm{kW})$ & 1.5 & 1.2 \\
PRF $(\mathrm{Hz})$ & $1-80000$ & $5000-20000$ \\
Pulsewidth (ns) & $200-2000$ & $250,500,1000$ \\
Noise figure $(\mathrm{dB})$ & 13 & 8 \\
Receiver bandwidth $(\mathrm{MHz})$ & 2,5 & $1,2,4$ \\
3-dB beamwidth $\left({ }^{\circ}\right)$ & 0.2 & 0.8 \\
\hline
\end{tabular}

that are contributing to the scientific archives of W-band radar observations, three $95-\mathrm{GHz}$ radars were deployed by the University of Massachusetts (UMass), The Pennsylvania State University (Penn State), and NASA's Jet Propulsion Laboratory (JPL) at the U.S. Department of Energy's Atmospheric Radiation Measurement (ARM) program field site in northern Oklahoma (ARM 1990) during summer 1998 (Sekelsky et al. 1999a). Systems from UMass and Penn State served as the ground-based sensors, while the $95-\mathrm{GHz}$ Airborne Cloud Radar (ACR), jointly developed by UMass and NASA/JPL, was installed in nadir-pointing mode on the NASA DC8 research aircraft. The ACR and the UMass Cloud Profiling Radar System (CPRS) were later redeployed in New Iberia, Louisiana, for coastal cloud and precipitation observation.

This paper first describes ACR and CPRS W-band radar calibration and intercomparison results. Next it presents a unique attenuation retrieval algorithm using dual-radar measurements. Finally, it compares the dualradar method to the Hitschfeld and Bordan (HB) algorithm (Hitschfeld and Bordan 1954). Data collected by CPRS and ACR from New Iberia, Louisiana, is analyzed using these algorithms.

\section{Radar systems}

The UMass CPRS is a portable ground-based, fully polarimeteric Doppler radar. It consists of W-band (94.92 GHz) and Ka-band (33.12 GHz) subsystems, a programmable pedestal to facilitate various scanning modes, a high-speed VXI-bus-based data acquisition system, and a digital signal processing (DSP) system. The construction of CPRS was funded by the U.S. Department of Energy (DOE) as a key remote sensor for cloud measurement under the ARM program. This paper concentrates on the use of the CPRS W-band radar [see Table 1 and Sekelsky and McIntosh (1996)] as a groundbased sensor and the combination of its measurements with airborne W-band radar data for atmospheric attenuation estimation.

The ACR (Table 1), jointly developed by UMass and JPL, is a compact airborne $95-\mathrm{GHz}$ polarimetric Doppler radar (Sadowy et al. 1997). It is distinct from previous
W-band cloud radars in that it incorporates new features, including an internal calibration loop, frequency agility, direct digital I and Q demodulation, and digital matched filtering in the receiver. It was designed as a research facility and serves as a prototype for development of the spaceborne cloud radar that will fly on CloudSat. During the past few years, ACR has been deployed on NASA's DC-8 and the DOE's Twin Otter aircraft in both upward-looking and downward-looking modes for various cloud experiments.

\section{Calibration and intercomparison}

The W-band radars are compact and highly portable. They are often installed in harsh environments on aircraft and in mobile ground-based systems that routinely move among remote field sites. System mobility and the delicate nature of W-band components may cause undetected calibration changes unless regular external calibrations are performed.

External calibrations are not trivial. The proper location, equipment, and supporting meteorological data are crucial for accuracy. They also require an instrumental configuration for the radar and calibration target that avoids reflections from unwanted clutter. Typically, the target is set atop a tower at some distance from the radar. The tower height is determined by the antenna beamwidth and its distance from the radar. Fortunately, millimeter-wave cloud radars have small antenna beamwidths, and the minimum height required for the corner reflector is low enough that it can easily be set atop a small tower. For example, a 1-m diameter Cassegrain antenna operating at $95 \mathrm{GHz}$ has a beamwidth of approximately $0.2^{\circ}$ implying a typical tower height and distance to the radar of 13 and $400 \mathrm{~m}$, respectively. Because the corner reflector data calibrates the system response to a known point target, the pulse shape and receiver frequency response must also be known accurately to apply the point target calibration to volume targets such as clouds (Doviak and Zrnić 1993; Sekelsky et al. 1999b). Finally, atmospheric absorption must be removed.

Following the summer 1998 DC-8 cloud radar experiment, both CPRS and ACR were set up at UMASS for external calibration using the same trihedral corner reflector. Subsequently, side-by-side cloud observations were made and compared with the calibration results. During these side-by-side intercomparisons, ACR and CPRS were both aimed in the zenith direction. The CPRS operated at a center frequency of $94.92 \mathrm{GHz}$ with a receiver bandwidth of $2 \mathrm{MHz}$, while ACR transmitted at $94.905 \mathrm{GHz}$ and had a receiver bandwidth of $2 \mathrm{MHz}$. The $15-\mathrm{MHz}$ frequency difference minimized interference between the 2 radars. A sample comparison of ACR and CPRS reflectivity profiles, which were obtained from observations of stratiform clouds with drizzle, is presented in Fig. 1. Since ACR's 3-dB antenna beamwidth is $0.8^{\circ}$, which is four times the $0.2^{\circ}$ beam- 


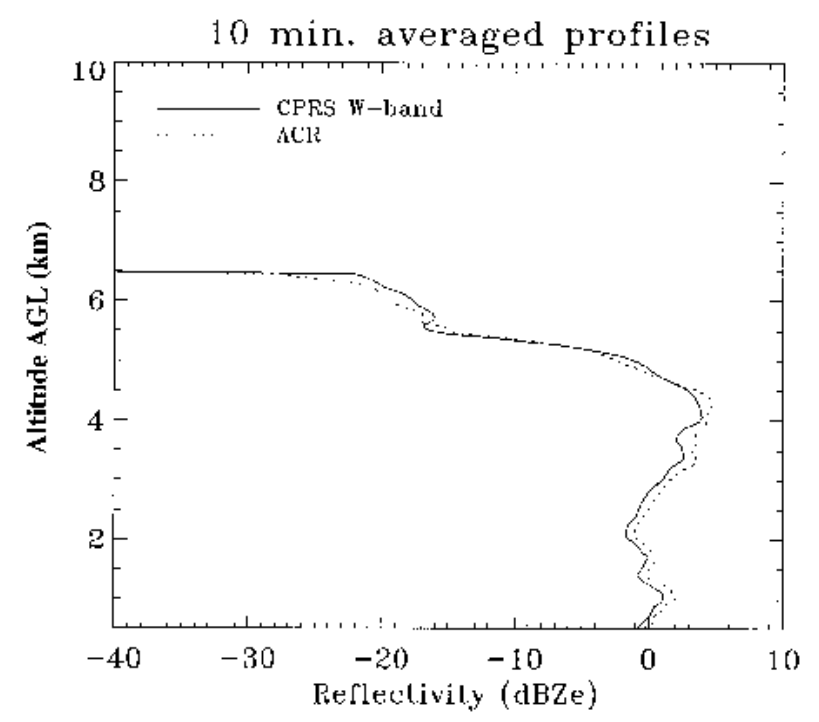

FIG. 1. ACR and CPRS reflectivities in low level stratiform cloud observations during the side-by-side intercomparison. A 10-min integration time is used in order to minimize the error.

width of the CPRS W-band radar, an integration time of 10 min was used for both radars in order to minimize the volume matching error. The mean difference between the ACR and CPRS radar reflectivity was found to be approximately $0.20 \mathrm{~dB}$.

A second approach was also used to verify the calibration of the airborne and ground-based systems. It took advantage of collocated airborne and ground-based radar reflectivity measurements of nonprecipitating clouds near New Iberia, Louisiana (Fig. 2). In order to match the sampling volume, 10 and $0.4 \mathrm{~s}$ averaging times were used for CPRS and ACR data, respectively. Using Liebe's atmospheric absorption model (Liebe $1985)$ and specific humidity data derived from local soundings, the measured reflectivity profiles in Fig. 2a were corrected for water vapor and oxygen absorption (Fig. 2b). Since the cloud base is approximately $7 \mathrm{~km}$ above the surface in this case, water vapor and oxygen cause significant attenuation of the ground-based signal while they have only a minor effect on the airborne radar measurements. After water vapor and oxygen attenuation are removed, the mean difference between CPRS and ACR reflectivity profiles is approximately $0.14 \mathrm{~dB}$.

While CPRS and ACR operate at slightly different center frequencies, there is some overlap in their transmitter bandwidths. This has the advantage of allowing precise timing of the ACR overpass of CPRS by the presence of each radar's transmitted signal in the other's receiver. In fact, this was used in real time to assess the correctness of the line being flown by the DC-8. A disadvantage is the contamination of potentially interesting data. For the analyses here, data at the precise overpass times are omitted, and the reported measurements are

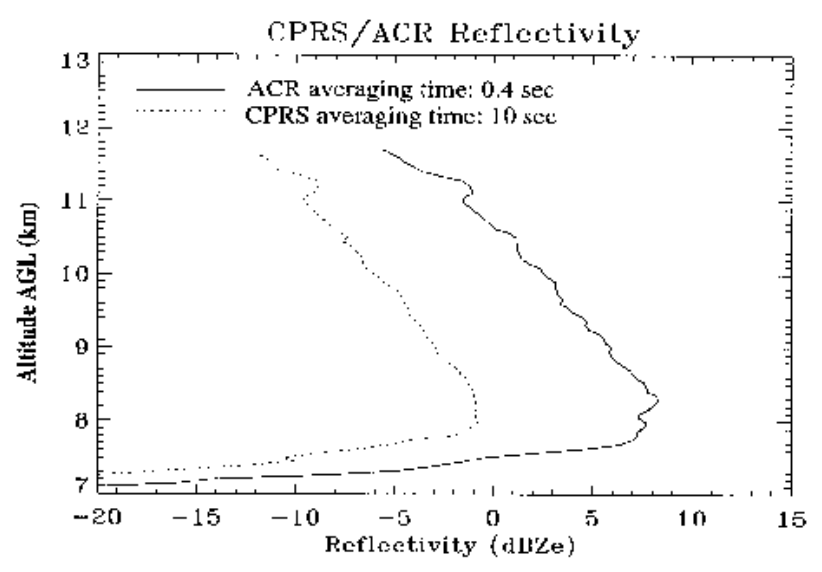

(a)

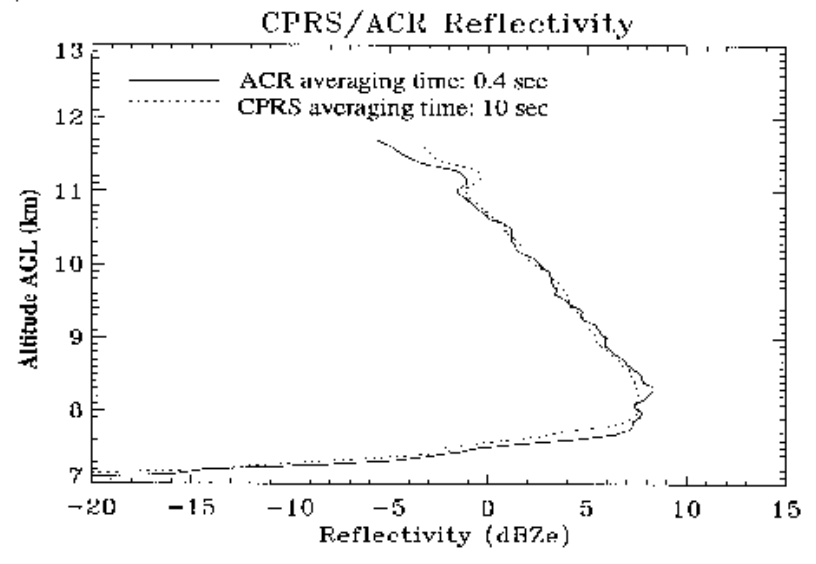

(b)

FIG. 2. Comparison of colocated airborne ACR and ground-based CPRS W-band radar profiles of nonprecipitating clouds (a) before and (b) after correction for water vapor and oxygen absorption. The mean of the difference between two profiles in (b) is $0.14 \mathrm{~dB}$.

for a time average of the data immediately before and after the omitted pulses.

\section{Attenuation retrieval algorithms}

This section describes two different attenuation retrieval algorithms. The first one is the dual radar method, which combines the collocated upward and downward radar measurements, while the second one is the HB algorithm, which uses single radar measurements with path-integrated attenuation as a constraint.

\section{a. Dual-radar method}

For radars operating at attenuating frequencies, the true equivalent reflectivity $Z_{e}$ is related to the measured reflectivity $Z_{e}^{m}$ in decibels by

$$
Z_{e}(n)=Z_{e}^{m}(n)+2 \sum_{i=0}^{n-1} k(i) \delta h,
$$




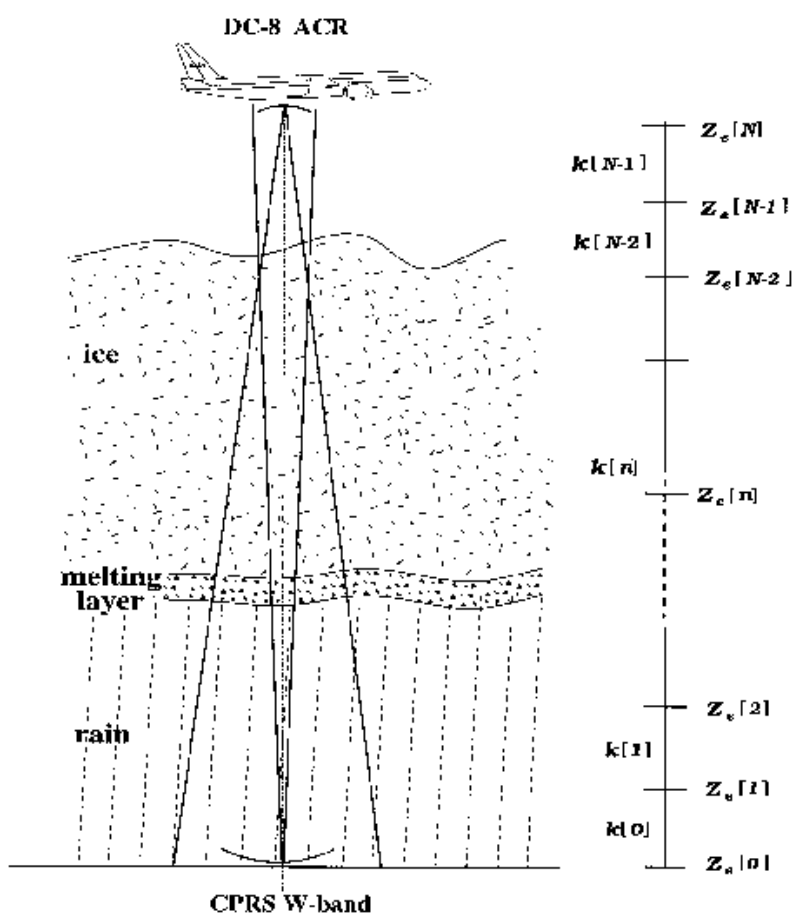

FIG. 3. Diagram of dual-radar measurement.

where $k=k_{a}+k_{p}+k_{c}$ is the total attenuation rate $(\mathrm{dB}$ $\left.\mathrm{km}^{-1}\right)$ due to atmospheric gases $\left(k_{a}\right)$, precipitation $\left(k_{p}\right)$, and clouds $\left(k_{c}\right) ; n$ is the radar range gate index; and $\delta h$ is the range sample spacing.

The dual-radar attenuation retrieval method uses measurements from opposing viewing angles to estimate attenuation as a function of range (Fig. 3). At range gate $n, Z_{e}(n), Z_{e, u}^{m}(n)$, and $Z_{e, d}^{m}(n)$ represent the true unattenuated reflectivity, reflectivity measured by the upwardlooking radar, and reflectivity measured by the downward-looking radar, respectively. For the upward-looking radar, we have

$$
\begin{aligned}
& Z_{e}(N)=Z_{e, u}^{m}(N)+A+C, \\
& Z_{e}(n)=Z_{e, u}^{m}(n)+2 \sum_{i=0}^{n-1} k(i) \delta h+C, \quad \text { and } \\
& Z_{e}(0)=Z_{e, u}^{m}(0)+C,
\end{aligned}
$$

which correspond to measurements at the top (range gate $N$ ), interior (range gate $n$ ), and bottom (range gate 0 ) of the atmospheric column under observation, respectively. The term $k[i]$ represents the total extinction rate at range gate $i$, and $C$ represents the wet radome attenuation, which affects the ground-based radar. The total two-way path-integrated attenuation, $A$, is given by

$$
A=2 \sum_{i=0}^{N-1} k(i) \delta h
$$

Similarly, the following equations apply to the downward-looking radar:

$$
\begin{aligned}
& Z_{e}(N)=Z_{e, d}^{m}(N), \\
& Z_{e}(n)=Z_{e, d}^{m}(n)+2 \sum_{i=n}^{N-1} k(i) \delta h, \quad \text { and } \\
& Z_{e}(0)=Z_{e, d}^{m}(0)+A .
\end{aligned}
$$

The following boundary conditions also apply at the top and bottom of the column of cloud and precipitation:

$$
\begin{aligned}
& C+A=Z_{e, d}^{m}(N)-Z_{e, u}^{m}(N), \quad \text { and } \\
& C-A=Z_{e, d}^{m}(0)-Z_{e, u}^{m}(0) .
\end{aligned}
$$

The wet radome attenuation, $C$, and total column attenuation, $A$, are derived from Eqs. (9) and (10). Substituting these values into (2)-(8) gives a set of $2 N+1$ simultaneous equations that can be solved for $k$ and $Z_{e}$ at each range gate

$$
\begin{aligned}
Z_{e}(n)= & {\left[Z_{e, d}^{m}(n)+Z_{e, u}^{m}(n)+A+C\right] / 2, } \\
k(n)= & {\left[Z_{e, d}^{m}(n+1)-Z_{e, d}^{m}(n)+Z_{e, u}^{m}(n)\right.} \\
& \left.-Z_{e, u}^{m}(n+1)\right] / 4 \delta h .
\end{aligned}
$$

The dual-radar algorithm has unique advantages over methods using data from a single radar. First, it provides both the total path attenuation and the attenuation rate at each range height. Second, a priori knowledge of the attenuation characteristics of atmospheric gases, clouds, and precipitation is not required, since radar reflectivity can be retrieved directly by combining the upward-looking and downward-looking reflectivity profiles. This significantly reduces the uncertainties in radar reflectivity estimation, since we do not have to make any assumptions about the microphysical properties of the hydrometeors. Once the radar reflectivity and attenuation are determined, a number of microphysical parameters of the target, such as liquid water content, ice water content, and precipitation rate, can be derived. Of course, this method can only be applied to simultaneous up/ down measurements.

\section{b. HB algorithm}

The equivalent radar reflectivity, $Z_{e}$, can also be retrieved from single-radar measurements if the relationship between $Z_{e}$ and $k$ is known. By using the powerlaw relation $k=a Z_{e}^{b}$, Hitschfeld and Bordan (Hitschfeld and Bordan (1954) developed an algorithm to correct attenuation errors in X-band radar reflectivity measurements, which in turn improved the accuracy of rain-rate estimation. According to the HB algorithm, (1) has an analytical solution for a downward-looking radar as follows:

$$
\begin{aligned}
Z_{e}(n)= & Z_{e}^{m}(n) \\
& -10 b^{-1} \log \left[C_{1}-0.2 \ln 10 a b \sum_{i=n}^{N-1} 10^{0.1 b Z_{e}^{m}(i)} \delta h\right] .
\end{aligned}
$$



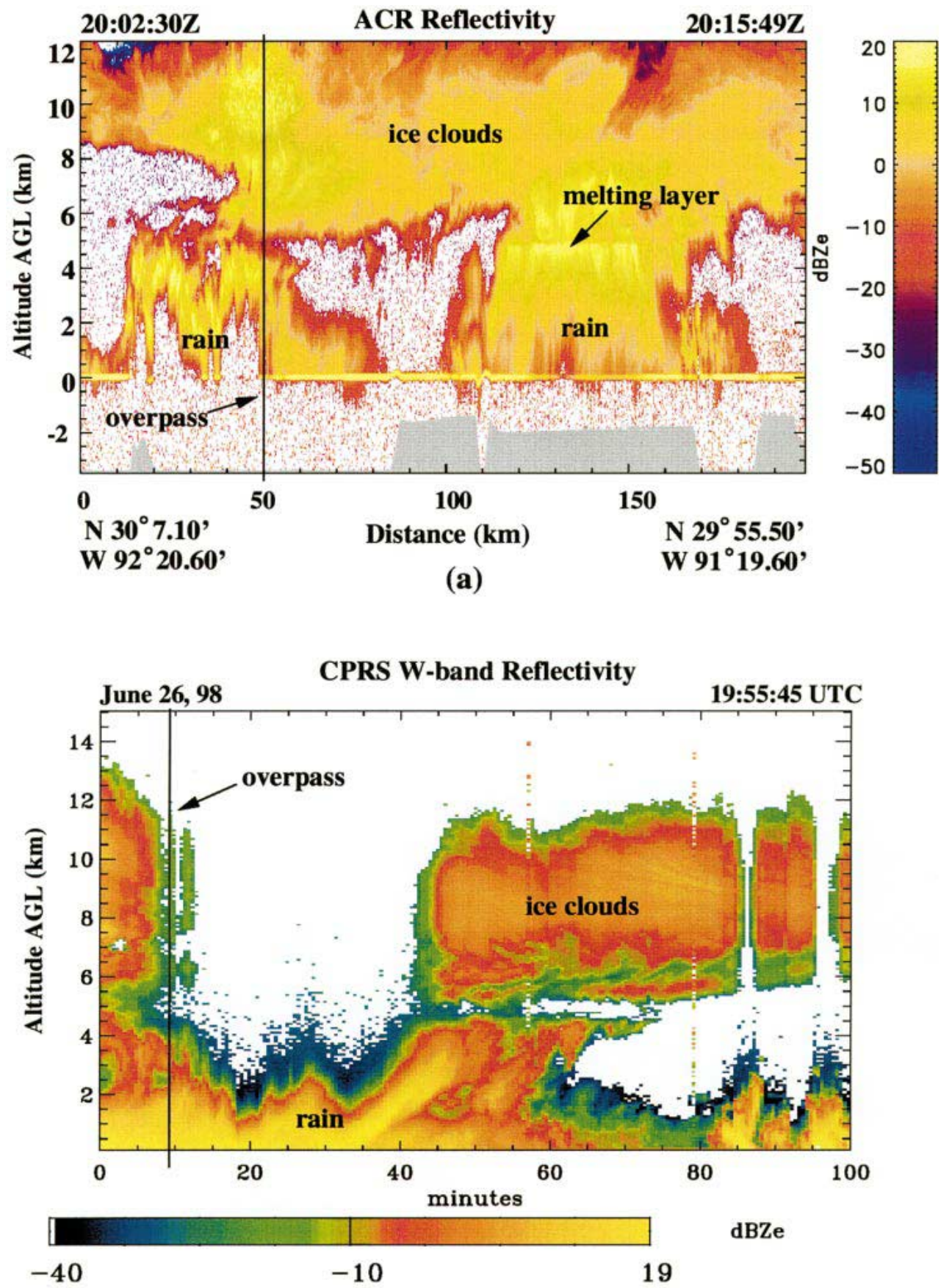

(b)

FIG. 4. (a) ACR and (b) CPRS W-band radar reflectivity images. The time when the DC-8 aircraft flew over the ground-based site is marked by a vertical line in each figure.

Here, it is assumed that $a$ and $b$ are constant with range. The terms $Z_{e}$ and $Z_{e}^{m}$ are in decibel scale, and $C_{1}$ is an arbitrary constant that is determined by a boundary condition. For instance, at the top of the column of cloud and precipitation, the downward-looking radar measures the unattenuated reflectivity, so $Z_{e}(N)=Z_{e}^{m}(N)$ and $C_{1}$
$=1$. Similarly, we can find the solution for an upwardlooking radar.

If the attenuation is small, the HB algorithm works reasonably well. When the attenuation is significant, however, the HB solution can become unstable due to errors in $k-Z_{e}$ relationships and radar calibration (Iguchi 


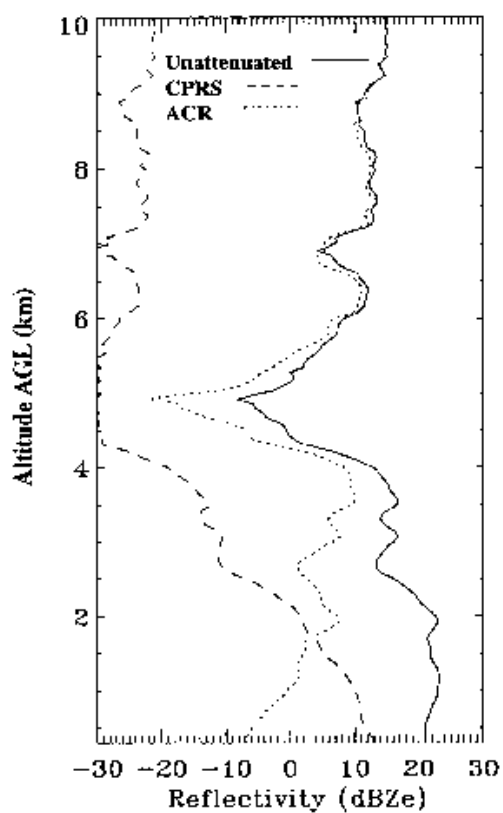

(a)

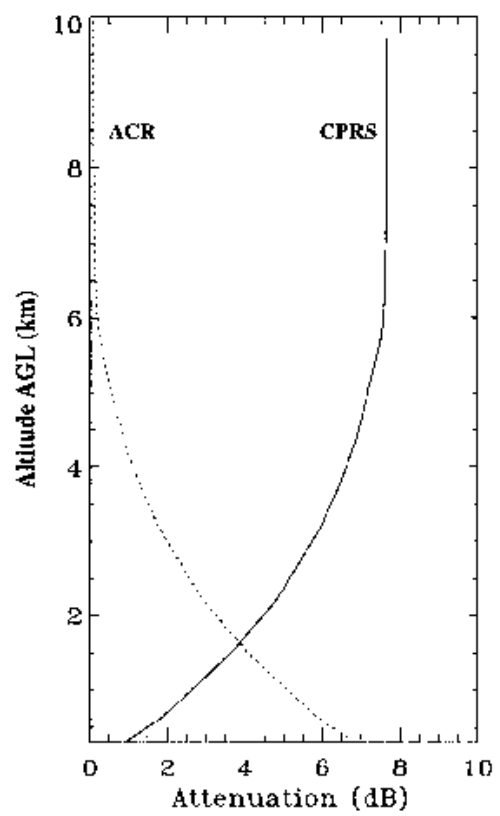

(b)

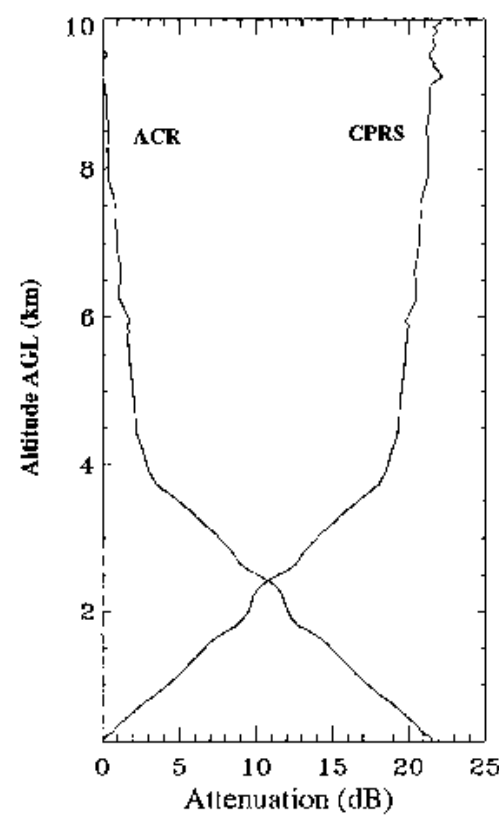

(c)

FIG. 5. (a) Radar reflectivity: the dashed and dotted lines represent CPRS W-band and ACR reflectivities, respectively, while the solid line represents the unattenuated reflectivity retrieved by combining ACR and CPRS W-band radar measurements. Regions above and below $5 \mathrm{~km}$ correspond to radar signal return from ice clouds and precipitation, respectively. (b) Two-way cumulative water vapor and oxygen attenuation for the airborne ACR and for the ground-based CPRS. (c) Two-way cumulative attenuation caused by rain and clouds, showing two distinct attenuation rates that are nearly constant with altitude. The precipitation region has an average two-way attenuation rate of 4.8 $\mathrm{dB} \mathrm{km}^{-1}$ while the ice clouds have a two-way attenuation rate of $0.38 \mathrm{~dB} \mathrm{~km}^{-1}$.

and Meneghini 1994). One way to solve these problems is to use path-integrated attenuation as a constraint. Path-integrated attenuation can be derived from radiometer measurements (Weinman et al. 1990) or from the surface reference technique (SRT) (Meneghini et al. 1983). It can also be derived from the dual-radar algorithm shown above. For a downward-looking radar, the two-way path-integrated attenuation is estimated from the radar measurement at the surface, $A=Z_{e}(0)$ $-Z_{e}^{m}(0)$. It can be expressed as

$A=10 b^{-1} \log \left[C_{1}-0.2 \ln 10 a b \varepsilon \sum_{i=0}^{N-1} 10^{0.1 b Z_{e}^{m}(i)} \delta h\right]^{-1}$,

where $\varepsilon$ is the correction factor for the $k-Z_{e}$ relation to allow equality in (14); (Meneghini et al. 1983). Since the radar signal experiences different extinction in liquid water clouds, in precipitation and in ice clouds, it is necessary to use different $k-Z_{e}$ relations for different hydrometeor regions if they are present in the path of the radar signal. In this case, path-integrated attenuation for these regions are obtained using the dual-radar measurements described above, and the HB algorithm is then applied to each region separately.

It should be noted that the HB algorithm cannot deal with water vapor absorption, since water vapor does not produce backscatter at millimeter-wave frequencies.
Therefore the power-law relation only accounts for the attenuation caused by precipitation or clouds, and attenuation due to atmospheric gases must be removed before using the $\mathrm{HB}$ algorithm.

\section{Experimental results}

During the day of 26 June 1998 in New Iberia, Louisiana, clouds with occasional showers and moderate rain caused by a tropical disturbance were moving over the southern Louisiana coastal area. One data case illustrates collocated measurements before moderate rainfall occurred. Figure 4 shows the CPRS and ACR reflectivity images. The vertical line in each figure marks the time when the DC-8 aircraft flew over the ground site. Figure 5a shows collocated radar reflectivity profiles. The dashed line and the dotted line are radar reflectivity measured by the CPRS W-band subsystem and by ACR, respectively. In order to improve signal-tonoise ratios and to reduce the volume mismatch between the two radar beams, CPRS W-band and ACR reflectivities are averaged for 30 and $0.4 \mathrm{~s}$, respectively. These averaging times were chosen based on radar beamwidths, aircraft motion, and wind speed. Using the dualradar algorithm described in section 4 , the true unattenuated radar reflectivity is obtained and shown by the solid line in Fig. 5a. At the surface, the difference between CPRS W-band measured reflectivity and the re- 


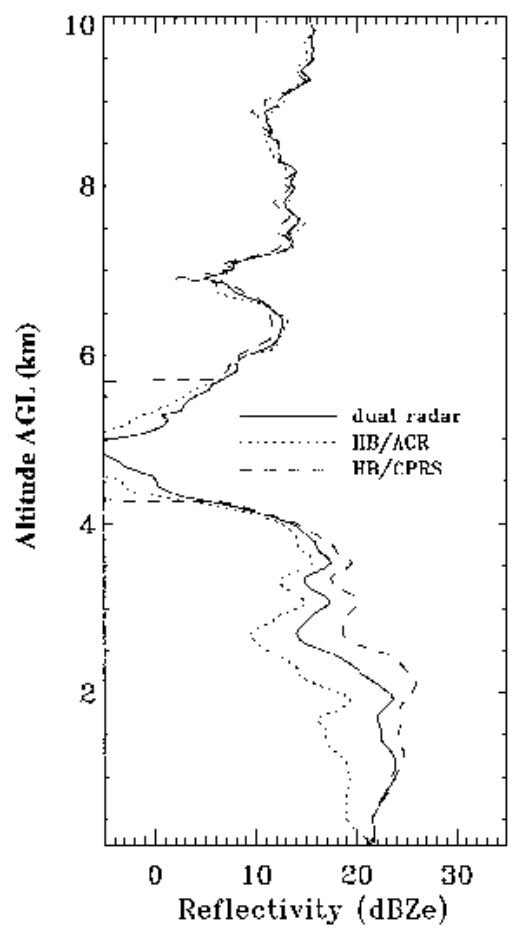

(a)

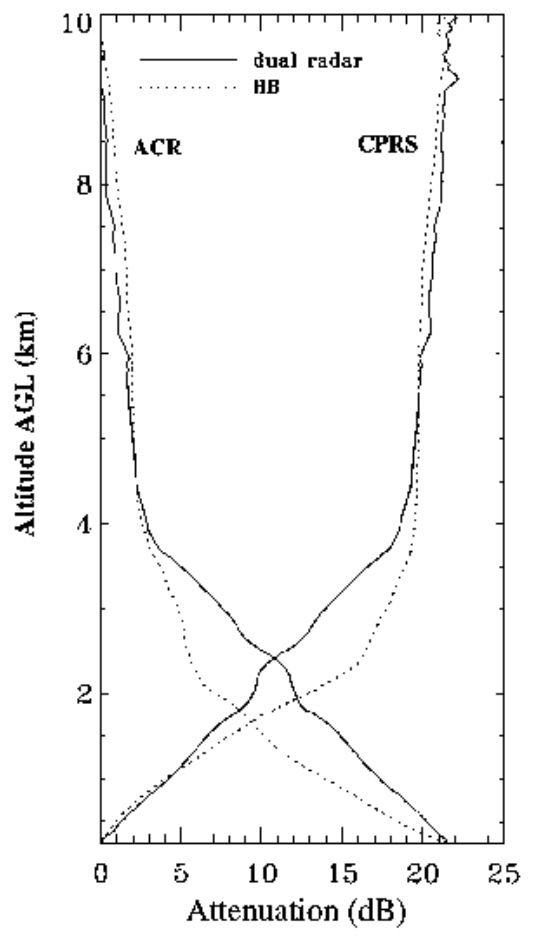

(b)

FIG. 6. (a) Retrieved radar reflectivities from the HB algorithm using uniform $k-Z_{e}$ relations within precipitation and ice cloud regions. The dotted line represents ACR reflectivity while the dashed line shows CPRS W-band reflectivity. The result from the dual-radar method is shown as a solid line. (b) Retrieved two-way path integrated attenuation along the radar beam path. The dotted curves are results from the $\mathrm{HB}$ algorithm using uniform $k-Z_{e}$ relations within precipitation and ice cloud regions, while the results from the dual-radar method are shown as solid curves.

trieved unattenuated reflectivity corresponds to the wet radome attenuation, $C$, which is $7.98 \mathrm{~dB}$ in this case.

While the dual radar method directly calculates total two-way extinction, extinction due to hydrometeors and gases can be separated, since gaseous attenuation can be calculated using local sounding data. The remaining attenuation is due to hydrometeor extinction. The curves in Fig. 5b show the 2-way cumulative attenuation caused by atmospheric gases, while Fig. 5c presents the 2-way cumulative hydrometeor attenuation, both corresponding to the data in Fig. 5a. Two distinct extinction rates, which are nearly constant with height, are shown in Fig. $5 \mathrm{c}$. The first region, for precipitation between the surface and $4 \mathrm{~km}$, has an average 2-way extinction rate of 4.8 $\mathrm{dB} \mathrm{km}^{-1}$. The second region in the ice cloud has an average 2-way extinction rate of $0.38 \mathrm{~dB} \mathrm{~km}^{-1}$. In this case, the total 2-way column extinction $A$ is $29.57 \mathrm{~dB}$, of which $7.6 \mathrm{~dB}$ is caused by atmospheric gases and $21.97 \mathrm{~dB}$ is due to cloud and precipitation extinction.

The HB algorithm is also applied to the same case for reflectivity and attenuation retrieval. Data from one of the radars is used as a single-radar measurement, while the data from the other radar is used to estimate the path-integrated attenuation. In the precipitation region, we take $k=0.022 Z_{e}^{1.13}\left(\mathrm{~dB} \mathrm{~km}^{-1}\right)$ derived by
Haddad et al. (1997), while $k=5.8 \times 10^{-3} Z_{e}^{0.98}(\mathrm{~dB}$ $\mathrm{km}^{-1}$ ) (Sassen and Liao 1996) is used for the ice cloud region. The retrieved reflectivity profiles are illustrated in Fig. 6a. For comparison, the unattenuated reflectivity retrieved by the dual-radar method is shown as a solid line in Fig. 6a. The total 2-way column extinction comparison is presented in Fig. 6b. From Fig. 5c, the 2-way path-integrated attenuation for the ice cloud is approximately $2 \mathrm{~dB}$. This yields a correction factor $\varepsilon$ in Eq. (14) of 2.68, which means the actual $k-Z_{e}$ relation for the ice cloud has a larger coefficient than the value given by Sassen and Liao (1996). Since Sassen and Liao's relation was developed using data from thin cirrus-like clouds, it may underestimate the attenuation in deep ice clouds as analyzed here. Further analysis has shown that most of the attenuation in the ice clouds studied here is due to scattering losses from large particles (Li et al. 2000).

The 2-way path-integrated attenuation for the precipitation region, as derived from dual-radar measurements, is $19.97 \mathrm{~dB}$. To satisfy the boundary conditions, values for $\varepsilon$ of 0.34 and 1.7 must be used for CPRS and ACR, respectively, in the precipitation region. The discrepancy between the results from the HB algorithm and the results from the dual-radar method is large. It should 


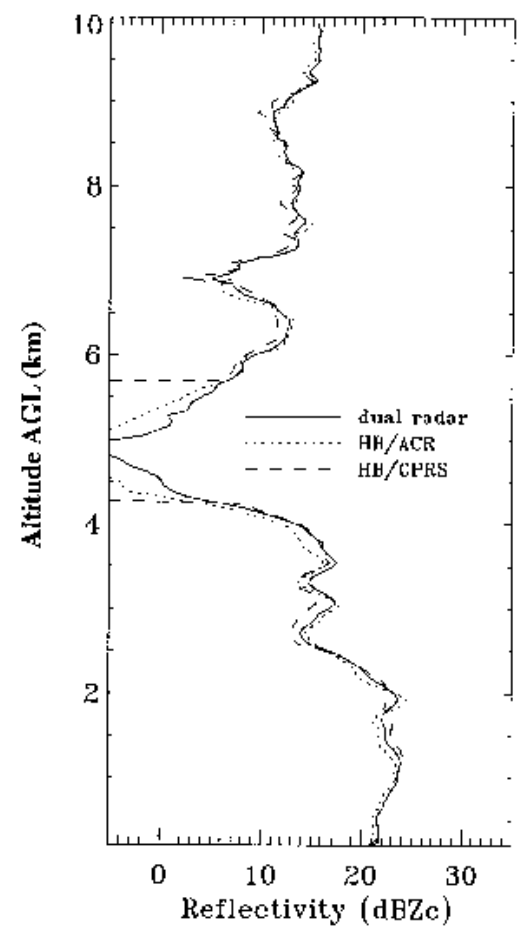

(a)

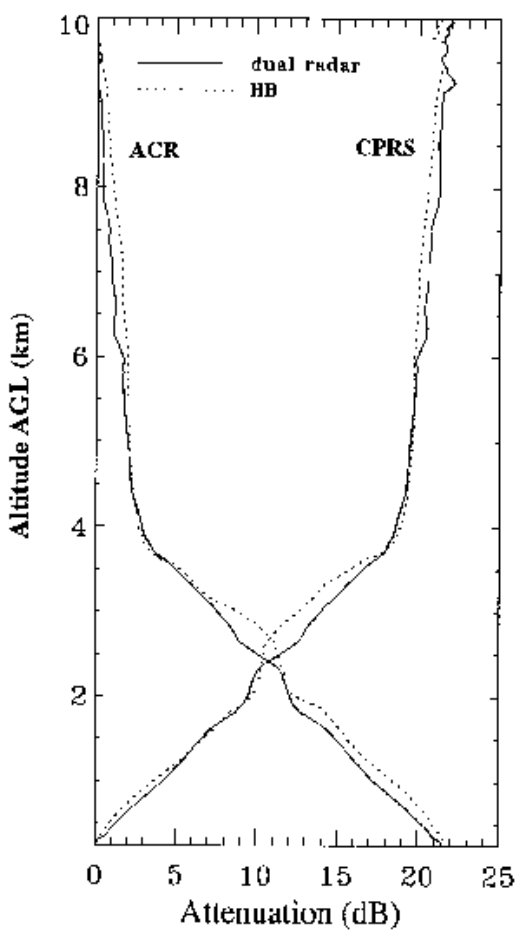

(b)

FIG. 7. Retrieved (a) radar reflectivity and (b) two-way path integrated attenuation along range using two different coefficients in the $k-Z_{e}$ relations within the precipitation region. Line types have the same meaning as those shown in Fig. 6.

be noted that in Fig. 5c, the extinction rate between the surface and $4 \mathrm{~km}$ is almost constant, but that in Fig. 5a, the unattenuated reflectivity retrieved by the dual radar method has 1 mean level from the surface to $2.3 \mathrm{~km}$ and another mean level between 2.3 and $4 \mathrm{~km}$. This suggests that different drop size distributions exist in those regions, and different coefficient values in the $k-$ $Z_{e}$ relations should be used. Consequently, the precipitation region was divided into 2 subregions. Region 1 is from the surface to $2.3 \mathrm{~km}$ with a $10.8 \mathrm{~dB}$ 2-way path-integrated attenuation, while region 2 is from 2.3 to $4 \mathrm{~km}$ with a $9.17 \mathrm{~dB}$ 2-way path-integrated attenuation. Different $k-Z_{e}$ coefficients are then used in region 1 and region 2, while a uniform $k-Z_{e}$ relationship is still used in the ice cloud region. Figures $7 \mathrm{a}$ and $7 \mathrm{~b}$ show the retrieved radar reflectivity and 2-way path-integrated attenuation along the path. This time, the $\varepsilon$ values are 0.32 for both CPRS and ACR in region 1 and 2.84 for both CPRS and ACR in region 2. With different $k-Z_{e}$ relationships, the results from the $\mathrm{HB}$ algorithm and from the dual-radar method show good agreement.

As shown above, the dual-radar method requires no knowledge of $k-Z_{e}$ relationships, but the HB algorithm does require proper $k-Z_{e}$ relationships for different hydrometeor regions to produce accurate retrievals. In most cases, assumptions have to be made in order to obtain these $k-Z_{e}$ relationships, and these assumptions introduce errors in reflectivity and attenuation estima- tion. In addition, accurate path-integrated attenuation estimation is also critical for using the HB algorithm when attenuation is large.

\section{Conclusions}

Radar reflectivity measurements of clouds are critical for determination of vertical profiles of cloud ice and liquid water content. One of the challenges of using millimeter-wave radars for quantitative measurements of clouds is attenuation caused by atmospheric gases and hydrometeors. Although attenuation is an unwanted effect, knowledge of radar signal path attenuation can be used to constrain algorithms that derive rain rate and other physical properties from high-frequency radars. This can be accomplished in several ways. The HB algorithm was developed for radar reflectivity and attenuation retrieval using measurements from a single radar. It requires an accurate estimation of path-integrated attenuation in order to yield proper results. The coefficient in the $k-Z_{e}$ relation should be adjusted for each distinct region if the cloud or precipitation region is not homogeneous along the radar beam path.

This paper also demonstrates a unique method for attenuation correction using two calibrated radar systems viewing a cloud volume from opposite viewing angles. This method does not require assumptions about the microphysical characteristics of the hydrometeors, 
thereby significantly reducing the uncertainty in radar reflectivity and attenuation estimation. Further analysis is planned to employ this algorithm in order to refine single-radar attenuation retrieval techniques, which will ultimately be used by operational sensors such as the CloudSat radar. In addition, once the CloudSat radar is operational and the ground track of the radar beam is known in advance, the ground-based radar will be deployed at proper locations to perform collocated measurements, and the dual-radar algorithm can be implemented for atmospheric attenuation retrieval.

Acknowledgments. The research was founded by the U. S. Department of Energy under Grant DE-FG0290ER61060 and by NASA's Jet Propulsion Laboratory under Contract 960108. Support from personnel at the Microwave Remote Sensing Laboratory at the University of Massachusetts and at the DOE Southern Great Plains (SGP) ARM site is gratefully acknowledged. The helpful comments of anonymous reviewers are appreciated.

\section{REFERENCES}

ARM, 1990: Atmospheric Radiation Measurement Program plan. U.S. Department of Energy DOE/ER-04441, Washington, DC, $116 \mathrm{pp}$.

Clothiaux, E. E., M. A. Miller, B. A. Albrecht, T. P. Ackerman, J. Verlinde, D. M. Babb, R. M. Peters, and W. J. Syrett, 1995: An evaluation of a 94-Ghz radar for radar remote sensing of cloud properties. J. Atmos. Oceanic Technol., 12, 201-229.

Doviak, R. J., and D. S. Zrnić, 1993: Doppler Radar and Weather Observations. 2d ed. Academic Press, $562 \mathrm{pp}$.

Haddad, Z. S., D. A. Short, S. L. Durden, E. Im, S. Hensley, M. B. Grable, and R. A. Black, 1997: A new parameterization of the rain drop size distribution. IEEE Trans. Geosci. Remote Sens., 35, 532-539.

Hitschfeld, W., and J. Bordan, 1954: Errors inherent in the radar measurements of rainfall at attenuating wavelengths. J. Meteor., 11, 58-67.

Houghton, J. T., L. G. Meira Filho, B. A. Callander, N. Harris, A. Katenberg, and K. Maskell, 1995: Climate Change 1995-The
Science of Climate Change. Cambridge University Press, 572 pp.

Iguchi, T., and R. Meneghini, 1994: Intercomparsion of single-frequency methods for retrieving a vertical rain profile from airborne or spaceborne radar data. J. Atmos. Oceanic Technol., 11, $1507-1516$

Lhermitte, R. M., 1987: A 94-GHz Doppler radar for cloud observations. J. Atmos. Oceanic Technol., 4, 36-48.

Li, F. K., E. Im, S. L. Durden, and W. J. Wilson, 1994: Airborne and spaceborne cloud radar designs. Proc. Int. Geoscience and Remote Sensing Symp. Pasadena, CA, IEEE, 672-674.

_- and Coauthors, 2000: Ice cloud extinction retrieval using ground-based and airborne millimeter-wave cloud radar measurements. Proc. 10th ARM Science Team Meeting, San Antonio, TX, Atmospheric Radiation Measurement Program, 1-6.

Liebe, H. J., 1985: An updated model for millimeter-wave propagation in moist air. Radio Sci., 20, 1069-1089.

Mead, J. B., A. L. Pazmany, S. M. Sekelsky, and R. E. McIntosh, 1994: Millimeter-wavelength radars for remotely sensing clouds and precipitation. Proc. IEEE, 82, 1891-1906.

Meneghini, R., J. Eckerman, and D. Atlas, 1983: Determination of rain rate from a spaceborne radar using measurements of total attenuation. IEEE Trans. Geosci. Remote Sens., 21, 34-43.

Pazmany, A. L., R. E. McIntosh, R. D. Kelly, and G. Vali, 1994: An airborne $95 \mathrm{GHz}$ dual polarization radar for cloud studies. IEEE Trans. Geosci. Remote Sens., 1, 731-739.

Sadowy, G. A., and Coauthors, 1997: The NASA DC-8 airborne cloud radar: Design and preliminary results. Proc. Int. Geoscience and Remote Sensing Symp. Singapore, IEEE, 1466-1469.

Sassen, K., and L. Liao, 1996: Estimation of cloud content by Wband radar. J. Appl. Meteor., 35, 932-938.

Sekelsky, S. M., and R. E. McIntosh, 1996: Cloud observations with a polarimetric $33 \mathrm{GHz}$ and $95 \mathrm{GHz}$ radar. Meteor. Atmos. Phys., 59, 123-140.

- L. Li, G. A. Sadowy, S. J. Durden, S. L. Dinardo, F. K. Li, A. C. Huffman, and G. L. Stephens, 1999a: Measurements of atmospheric extinction using combined airborne and ground-based $95 \mathrm{GHz}$ radar observations. Proc. Int. Geoscience and Remote Sensing Symp., Hamburg, Germany, IEEE, 461-463.

- , and Coauthors, 1999b: Radar calibration validation for the SGP CART Summer 1998 DC-8 Cloud Radar Experiment. Proc. Ninth ARM Science Team Meeting, San Antonio, TX, Atmos. Radiation Measurement Program, 1-5.

Weinman, J. A., R. Meneghini, and K. Nakamura, 1990: Retrieval of precipitation profiles from airborne radar and passive radiometer measurements: Comparison with dual-frequency radar measurements. J. Appl. Meteor., 29, 981-993. 